\title{
Organisational Factors Associated With Readiness to Implement a Preventive Intervention for Overweight and Obese Patients in Australian General Practice: a Mixed Methods Exploration From the HELP GP Trial.
}

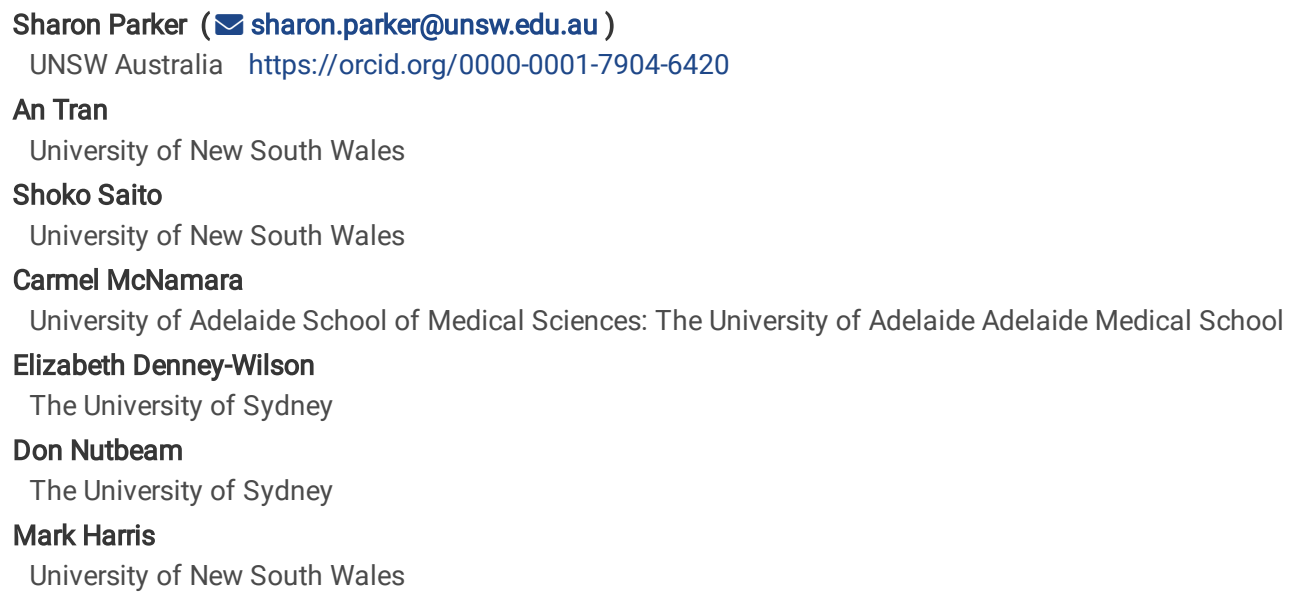




\section{Abstract}

\section{Background:}

Australian rates of obesity are increasing. General practice has a definite role to play in addressing obesity and preventing further chronic disease but relatively few encounters focus primarily on weight management. The HeLP GP trial assisted overweight and obese patients to make positive lifestyle changes to diet and physical activity and increase the capacity of practice nurses to deliver comprehensive weight management. The nurse led intervention comprised a health check and access to a lifestyle app and/or telephone coaching. Within this paper we describe the experience of implementing this intervention through the lens of organisational readiness with specific emphasis on the role of the practice nurse.

\section{Methods:}

Routinely collected research data, quantitative practice surveys, and qualitative field notes from research officers and facilitators were mapped against a recognised organisational readiness framework. Motivation to implement, general capacity and intervention-specific capacity were assessed.

\section{Results:}

The level of organisational readiness within the participating practices was observed to vary considerably, particularly the domain of intervention specific capacity. The level of practice nurse turnover negatively impacted the implementation of the intervention, affecting half of the intervention practices. Within this study we observed a general lack of practice-based support for the practice nurse regarding intervention delivery and varying levels of interest, skill, and confidence in delivering the program to patients. Nurses struggled to complete the health checks and subsequently to conduct timely follow up appointments Risk assessment and referral to telephone coaching were generally not problematic, although we noted lower confidence with the setup of the lifestyle app and with instructing patients to use it.

\section{Conclusions:}

Using an organisational readiness checklist is valuable for determining specific capacity issues prior to commencing research but should also assess the capacity of those individuals responsible for the intervention. We found a lack of general 'readiness' inherent in the practice nurse role. If they are to fulfil their potential in supporting patients to reduce risk and adopt healthier life choices, our study indicates that more could be done to improve their workforce positioning and remuneration, which may, in turn, improve continuity of care, retention, and individual motivation.

\section{Trial registration:}

The HeLP GP trial is registered with the Australian and New Zealand Clinical Trials Registry (ANZCTR - ACTRN12617001508369). The trial was registered 26/10/2017 http://www.ANZCTR.org.au/ACTRN12617001508369.aspx

\section{Background}

Obesity rates have tripled worldwide in the last 40 years and represent a significant public health challenge(1). Within Australia, $63 \%$ of the population are overweight or obese (Body Mass Index (BMI) $25 \mathrm{~kg} / \mathrm{m} 2$ or more) (2), contributing to the chronic disease burden (3) and $8 \%$ of overall disease burden within lower socioeconomic groups (4). Socioeconomic differences in BMI among adults substantially contribute to avoidable deaths and direct healthcare costs (5).

Although obesity prevalence is increasing among patients attending general practice (3), less than $1 \%$ of general practice encounters are documented by General Practitioners (GPs) as being primarily focused on obesity (6). Barriers to providing effective obesity management in this setting include time constraints, lack of adequate training, frustration at previous outcomes, poor perceived patient motivation (7), and a pervasive belief among some clinicians that discussing weight is inappropriate, unfeasible or unacceptable to patients (8).

Overweight and obesity is influenced by a range of physical, economic, political, and sociocultural factors that interact to produce an obesogenic environment (one which promotes obesity in individuals and populations) $(4,9)$. While primary care interventions cannot address all these factors, it can make a positive contribution through individual risk assessment and identification, and the promotion of lifestyle interventions which emphase healthy eating, increased physical activity and behaviour change $(10,11)$. Evidence based weight management programs utilising practice nurses (PNs) have shown some positive results in terms of patient outcomes and cost (12), and have been shown to be feasible, acceptable and valuable in the Australian setting (13) although not currently funded through Medicare (the Australian universal health scheme).

Despite progress in developing and testing primary care interventions to support weight management, relatively little is known about the practicality of implementing and sustaining potentially effective interventions. Implementation research within general practice is relatively uncommon but is essential to ensure the broader application of effective obesity interventions. When evaluating such interventions, it is not only important to assess the outcomes but also the environment in which the intervention was introduced (perceived affordability, the capacity of the organisation and individuals to accept and initiate change, to redesign their systems or their usual behaviours, adjust staffing roles, alter workflow, decision making and communication).

The concept of "organisational readiness" is now much better understood as important in determining whether a new policy, program or practice can be successfully implemented in a healthcare setting (14). Implementing something new can be challenging and often requires coordinating change among multiple aspects of a practice setting (15). When organisational readiness is high, members are more likely to initiate change, exert greater effort in support of change, and exhibit greater persistence in the face of obstacles or setbacks resulting in greater consistency and quality of intervention delivery (16). 
This paper describes the experience of implementing a nurse led obesity intervention in Australian general practice through the lens of organisational readiness. Drawing upon a sub-set of data collected in the HeLP GP trial (17) we describe key organisational characteristics of participating practices including capacity, capability and motivation in order to contextualise organisational readiness for this specific intervention, with emphasis on the PN role.

\section{Context}

A cluster randomised design was used with general practices[1] in two Australian states; New South Wales (NSW) and South Australia (SA)[2]. Practices were selected based on their geographical location: (Local Government Areas LGAs) with a Socio-Economic Indexes for Areas (SEIFA)(18) equal to and below the $8^{\text {th }}$ decile)[3] and stratified according to the size of the practice and the state to ensure equal allocation. Patient eligibility included a Body Mass Index (BMI) $\geq 28$, aged between 40-74 years, having weight and blood pressure (BP) recorded in the last 12 months, speaking English and/or Arabic, Chinese or Vietnamese and access to a smartphone or tablet. Patients were excluded if they had experienced $>5 \%$ weight loss in the past three months, were taking weight loss medications, or had diagnosed chronic illnesses (diabetes requiring insulin, cardiovascular disease, serious mental illness), or cognitive or physical impairment prohibiting participation in moderate level physical activity (17).

The intervention was a PN led model of health assessment combined with mHealth (mysnapp lifestyle app) and telephone coaching (Get Healthy). Access to the app and the coaching were facilitated by the PNs and both the PNs and GPs conducted patient follow up ( 6 and 12 weeks respectively) (Figure 1 ). The trial sought to help overweight or obese patients to make positive lifestyle changes to diet and physical activity and to assess the value, sustainability, and scalability of this nurse-led model.

Control practices provided the usual care offered to their patients with overweight or obesity.

\section{a) Practice recruitment}

During 2018, 22 practices were recruited to participate (11 intervention and 11 control). Eight intervention practices were from Sydney (NSW) and three were from Adelaide (SA). Research centres at The University of Adelaide (UoA) and the University of New South Wales (UNSW) managed recruitment with the assistance of the local state Primary Health Network (PHN)[4]. One Sydney intervention practice subsequently withdrew at the point of patient recruitment.

\section{b) What was expected of the participating practices?}

All practices, regardless of allocation, were required to install the trial software (Doctors Control Panel (DCP), and to recruit patients. We required consent from at least one GP and one PN and the participation of reception staff to distribute documentation to potentially eligible ('flagged') patients at the point of presentation. The GP/PN was not responsible for consenting patients but they could conduct basic eligibility screening and discuss the trial with their patients. Interested patients could leave their consent at a secure collection point within the practice or return it to the coordinating centre (UNSW) in a replypaid envelope.

\section{c) What was expected of the intervention PNs and GPs?}

Since 2012 funding has been provided to Australian General Practice to increase the number of PNs, to expand their role, promote collaborative and team based care, and to engage PNs in clinical and preventive care (19). PNs had a pivotal role in the HeLP GP intervention (Table 1). Through a shared portal in DCP, PNs and researchers could communicate regarding patient encounters, issues of eligibility and non-attendance, and schedule dates for trial events. PNs were expected to arrange patient appointments (3 contact attempts), document these within DCP and update clinical changes in the patient's medical record to enable accurate extraction of trial data. PNs were provided 3 on-line training modules, developed specifically for the Help-GP trial to assist them with intervention tasks.

GPs were asked to complete one on-line training module (15 minutes) and encouraged to conduct a 12-week patient follow up to review the patient's BMI, waist circumference (WC) and blood pressure (BP), to briefly discuss the patient's experience of mysnapp and Get Healthy, and to devise a 12-month weight management plan. PNs and GPs completed a pre/post survey outlining their demographic details and their attitudes to their preventive clinical work, plus a profile of their practice (staffing, software, recall of patients and use of patient resources).

\section{Methods}

We used a mixed method research design. During implementation of the intervention, a research officer (RO) in each state conducted weekly/fortnightly visits to all practices for data collection, to troubleshoot issues, provide information and support to PNs and other staff, and to collect clinical data and provider feedback. Each RO documented their field experiences and observations including their impression of the practice environment (culture and routines), the feedback from intervention staff, and the interaction between practice staff and trial processes and systems. Additionally, a facilitator in each state documented each of the three formal facilitation sessions held with PNs, designed to support, and guide them in intervention delivery. These sessions promoted uptake through education on risk factor recording and promoting work practices (scheduling of reminders for the health check and follow up, use of the lifestyle app and referral to the Get Healthy service). Facilitators also documented their observations about aspects of practice and PN engagement with the intervention.

Additional quantitative data from baseline provider surveys (GPs and PNs) and practice profile surveys (baseline only) were analysed using SPSS. Descriptive process indicators for the PN health check and follow up (timing, and completion) were tabulated descriptively from the DCP data using Excel.

Integrated qualitative and quantitative data sources were used to assess each intervention practice against indicators related to three specific organisational readiness domains (Table 2): motivation to implement, general capacity and intervention-specific capacity using a recognised framework (20). This was done 
during a workshop involving the RO's, a facilitator and the trial coordinator. Joint discussion was used to map individual practice data to each component, thereby allowing for visual tabulation and comparison between practices. Each component was classified as having a positive $(* \star \star)$ or negative $(*)$ impact. Survey and DCP data were used where this supported an understanding around some framework domains, specifically items related to workforce, organisational capacity, and intervention specific capacity. Organisational readiness was not measured as a proxy for individual practice performance (outcomes of care or efficiency) but was used to identify the factors impacting intervention implementation and to provide a more complete picture of the complex contextual factors impacting the research.

\section{Results}

We used the Good Reporting of a Mixed Methods Study (GRAMMS) checklist to guide the reporting in this article (21).

\section{Practice Characteristics}

Table 3 provides an overview of the baseline characteristics of all practices. Overall, $35 \%$ of available GPs and $80 \%$ of available PNs from the participating practices agreed to participate. There was no difference in the proportion of GPs and PNs participating by allocation.

\section{Characteristics of the providers}

Table 4 provides self-reported baseline provider characteristics. There was an even gender mix among GPs but PNs were predominantly female (92\%) and predominantly younger with $54 \%$ being aged between $20-34$-years. Among the PNs, $65 \%$ worked part time whereas $85 \%$ of GPs worked full time. Compared to GPs, PNs had worked fewer years in general practice (mean 4 years) and less years in their current practice (mean 2 years). Almost half of the GPS and PNs reported that they never or rarely used apps or websites with their patients. PNs were more likely than GPs to indicate they were moderately/very confident to show patients how to use apps (51\% versus $22 \%)$.

\section{Organisational Readiness domains}

Table 5 displays assessment of the organisational readiness domains for each intervention practice. The one NSW practice that did not recruit any patients has not been included in the discussion of intervention specific capacity.

\section{a) Motivation}

Due to a targeted practice recruitment of those interested in prevention and research, we can assume some level of motivation on the part of practices to participate and implement the HeLP GP intervention. Incentives were also provided in the form of one-off monetary practice payment for participation (AUD $\$ 1,000$ ), completion of PN health checks (AUD \$40 per patient) and follow up (AUD \$20 per patient). Continuing Professional Development (CPD) activities were also provided for GPs and PNs which contributed towards meeting professional educational criteria.

Analysis of the recorded observations from the ROs and facilitators working with the practices revealed that four practices displayed strong commitment to providing good preventive care and/or a belief that the practice staff should contribute to research. Increasing numbers of overweight patients within practices was also a common reason why practices wanted to instigate a weight management intervention with their patients. For one GP, a recent close working relationship with the organising centre (UNSW) and a strong interest in obesity drove the decision to participate.

All except one practice indicated at the outset that the intervention would be achievable, although the time commitment required by PNs raised concerns for some. In one smaller Sydney practice where the GP worked across two sites, and the PN worked part-time, reluctance was observed on the part of the PN because the GP had agreed to participate while she was on leave. Despite generally good motivation levels initially, these were observed to decline in some practices over time. This was not a short intervention and a 12 -14-month commitment was required by practices for set up, training, patient recruitment, intervention delivery and data collection. RO's and facilitators described a steady loss of motivation over time due to various factors including work pressures, inadequate or changes in staffing, organisational barriers, and competing clinical priorities such as general practice accreditation and a busy influenza season. For one rapidly expanding practice, motivation decreased as the practice became busier and pre-occupied with mounting organisational issues.

\section{b) General Organisational Capacity}

\section{Climate/Culture}

Multiple and varied practice structures were observed among the participating practices. Of note were reported cases where hierarchical, haphazard, or disorganised internal systems reduced staff participation in decision making, particularly around involvement in the trial. Most practices displayed a 'top-down approach' where decisions were made by the GP/s resulting in situations where other practice staff felt they had limited opportunity to contribute to decision making, and hence limited opportunity to initiate or influence changes within the practice. Only in one SA practice were the GP, PN and Practice Manager (PM) observed to equally influence the decision about participation. Here, the PN was pro-active and clearly supported by the GP, indicating mutual trust and respect within the relationship. Conversely, reception staff at this practice were not engaged in decision making despite being tasked with the distribution of trial materials to potentially eligible patients.

Despite a general tendency for PNs in this study to lack decision making capacity within the practice, they seemed consistently autonomous in their day-today clinical work. GPs were not reported to micro-manage or monitor the PNs delivery of, or the involvement of other staff in the HeLP GP intervention. ROs and facilitators reported that GPs were frequently unaware as to whether the reception staff or PNs were actively undertaking the trial tasks or completing 
them according to trial protocols. Coordinating roles were sometimes undertaken by PMs. In other cases, PNs coordinated the reception staff and the GP to complete tasks, but this was specific to a few practices only.

\section{Staffing and Resources}

No initial difficulties relating to practice space, equipment issues or staffing quotas were identified. Practice eligibility required at least one PN to implement the intervention, but this was sometimes shared across two positions. Consistent and widespread PN turnover however significantly impacted the capacity to implement the Help GP intervention. As PNs infrequently provided prior notification to the trial that they were leaving, we were unable to elicit whether this level of turnover represented dissatisfaction with their employment, or some other work related or personal trigger. Both study groups experienced substantial turnover, the intervention group recording slightly higher rates than the control group (11 versus 8 PNs). PN turnover, however, affected half of all intervention practices at some point and was a persistent disruptive element within the trial. At each occurrence, RO's had to re-commence orientation and training, and facilitators had to repeat support visits. It was also challenging for the new PNs to pick up the role part way through the trial; they did not always feel confident with the research tasks, did not have existing rapport with the patients, and were sometimes unhappy with the unexpected workload or role. Consequently, the level of engagement of these 'replacement' nurses varied, and extra work on the part of the researchers was essential to achieve completion in the practices with consistent turnover. In addition, general workload was a major factor particularly for those PNs who were undertaking other research activities (1 practice) and those working reduced hours or sharing roles.

\section{Leadership and Communication}

The degree of practice leadership observed was variable and often linked to the attributes of individuals rather than a practice-wide norm. The level of leadership within each practice was also observed to be influenced by many factors, notably staffing arrangements and the consistency or fluctuation of staffing levels.

Difficulties establishing good lines of communication was observed by the ROs and facilitators. The primary contact in a practice could be the GP, the PM, the $\mathrm{PN}$, or a combination of these. In some cases, contact with the reception staff could be done directly, whereas in others, it was only via one of the other staff members. Particularly difficult were those circumstances where the primary contact could not be reached easily (e.g., a message had to be left for the GP), where the primary contact worked part-time/weekends, and in practices where inter office communication was disorganised. It was crucial to this research to identify the main contact person at each practice and to build individual relationships and develop tailored methods of interacting. Identifying this person, however, took time, and required considerable patience and flexibility. In some practices the reception staff were consistent, in others, there were multiple receptionists working different shifts, or more than one receptionist working at a time. Lines of responsibility and accountability were frequently vague or hard to discern.

\section{a) Intervention specific capacity}

\section{The PN Model - skills knowledge and ability to deliver the HeLP GP Intervention}

The HeLP GP intervention had three components that PNs could offer to their patients. Feedback and observation indicated that PNs were comfortable with the clinical content of the health check as this largely aligned with their normal work. Despite this, ROs and facilitators experienced significant ambivalence on the part of the PNs to conduct the health checks, noting a mix of disinterest, reluctance, and a lack of confidence by PNs to engage with their patients throughout the intervention. On-line training appeared well received, providing obesity education and tools and instruction to implement the intervention. PNs however, demonstrated variable success with delivering the health checks to consenting patients. One Sydney practice failed to successfully recruit any patients and hence no health checks were conducted at this site. In total 84/120 (70\%) consenting patients underwent a health check and completed the baseline assessment. At the point of health check, 16 patients withdrew, 14 patients could not be contacted after three attempts by the PN, and 3 patients were found to be ineligible. The health check was not completed for 2 patients who received insufficient follow up by the PN and 1 patient who was too busy to attend. Similarly, PNs struggled to complete the patient follow-up with 58/84 (69\%) patients being seen for the 6-week review (Figure 2). The mean number of days between the health check and the 6-week follow up was 64.2 days (range 42-199 days) indicating that this was frequently provided outside the designated timeframe (42 days).

The remaining components of the intervention (referral to Get Healthy and (mysnapp) required initiation of the health check and were therefore impacted when the health check was not conducted. Referral to Get Healthy could be initiated by phone, fax, or email and did not itself present a barrier for PNs. However, PNs demonstrated great variability in their skills and confidence relating to the introduction and set up of mysnapp. At baseline, almost half of the PNs had reported that they never or rarely used apps or websites with their patients, although PNs were more likely than GPs to say they were moderately or very confident showing patients how to use apps (51\% versus $22 \%$ ). In the individual cases where PN confidence was identified as a factor, additional facilitation was provided to them to encourage maximum uptake.

Within one Sydney practice, the PN model was supplemented by the employment of casual nurses when practice accreditation was prioritised over the intervention, and the PN was removed to that role. To avoid delays, the trial employed two casual PNs specifically to complete the patient health checks. We subsequently also offered this alternative to other practices who were struggling with the completion of health checks, but the offer was not taken up.

\section{Practice factors affecting the implementation of the HeLP GP intervention}

General support for the trial and the intervention ranged from welcoming, helpful, and interested to disinterested and in some cases oppositional. 'Push back' by reception staff was not widespread but was identified as a prominent issue in some practices and proved challenging to manage. As RO's were 'outside' the practice, they were unable to influence these dynamics and relied on goodwill and rapport building to negate these problems. Some receptionists resented the 
extra workload that resulted from printing documents, handing these to patients, and managing DCP. In some instances, the primary contact at the practice had no supervisory role over reception staff and, consequently, resistive behaviour remained throughout the trial.

Our experience was that practices uniformly required a great deal more RO support to carry out the required tasks than anticipated. Fortnightly visits were made to each practice through the patient recruitment and intervention period, as well as interim email and telephone support to try and maintain practice staff interest and engagement. Many practices required constant reminders and prompting about tasks and reinforcement about the reasoning for these tasks. Lack of time and workload were frequently cited as reasons for why trial tasks went undone. We did not identify a clear program 'champion' at any of the practices (i.e., someone who actively supported the intervention and provided continuous leadership). Although we identified some individuals who had a stronger interest in the intervention and responded in a timelier way, we did not identify clear standout people (or roles) within the practices who were prepared to advocate or promote significant changes, either to facilitate the intervention or because of the intervention. In at least half of the practices, the intervention was generally supported by the practice management (usually the GP/s) however this did not appear to translate to the PNs feeling supported to undertake the intervention. We identified $6 / 10$ practices where PNs expressed that they felt insufficiently supported from within the practice to undertake the intervention with their patients.

\section{Uptake and appreciation for trial systems and resources}

An extensive list of resources was developed for the trial and provided in paper, digital, and on-line modes (Table 6). It was not possible to monitor the uptake and use of all resources but the provider's website containing trial information to support processes and mechanisms for feedback, was generally poorly utilised. While some PNs were enthusiastic about the training, the completion of all three modules proved onerous for some PNs despite being in a format that could be done at a time that was convenient, and which could be used to apply for professional and educational recognition. PNs were not paid to undertake the training.

Over the trial period, most practices experienced some difficulties with the DCP software (used to identify eligible patients and monitor intervention progress). The nature of these issues was widespread and were ongoing for some practices. We cannot therefore discount the negative impact of this disruption, or the negative sentiment generated because of this disruption. Some practices managed their software systems in-house, but many used external IT management companies which added to the complexity and time involved to identify and address system issues. External IT support services sometimes felt uncomfortable about having the trial software installed or were reluctant to support technical issues such as firewalls. Also notable were issues with internet connection and speed which proved particularly problematic for PNs when trying to set up mysnapp for patients. We had anticipated difficulties with the app set-up and had provided troubleshooting documents and a reference video, but we don't know the extent to which these were utilised.

Practice nurses appreciated the guidance and support provided through the facilitation visits. We envisaged a total of three one-hour facilitation visits to each practice to address requirements and to answer questions. On average 4.7 visits were required to each practice by the facilitator (4.5 visits NSW; 5.3 visits SA). Facilitation number two (the visit where the PNs were briefed around the intervention), took an average of 98 minutes. This extra time was a due to a combination of higher PN need (unfamiliarity with apps, uploading health check templates and training to use trial software) and repeat visits due to PN turnover. Distance of some practices from the research centre resulted in just over 5 hrs per practice of travel time (Table 7).

\section{Discussion}

Using the lens of organisational readiness has provided a valuable opportunity to reflect on those organisational factors influencing this PN led obesity intervention. Despite the natural variation in the size, workforce, context and capacity of Australian general practices (22), we identified common organisational elements enabling us to present broad observations and suggestions regarding this model of patient care, and the level of organisational readiness required for this type of nurse led activity. Although we noted variation across all domains of the organisational readiness framework, it was the domain of intervention specific capacity where we noted the largest variation among practices.

Practice interest in this trial was largely driven by the ability to provide an in-house weight management program in response to growing numbers of overweight and obese patients. Although we can assume some general motivation and interest in participation, the HeLP GP intervention was not highly prioritised by practices, and the need to follow protocol, collect data and work within timeframes was often found to be overwhelming and/or of low priority. We acknowledge that difficulties experienced with trial software may have contributed to frustration and negative attitudes among some PNs and receptionists, and the length of the trial may have resulted in some research fatigue.

The turnover of PNs was much higher and widespread than anticipated and severely impacted the trial. PN turnover affected both groups within the trial with half of all PNs who initially consented leaving at some point. Substantial problems of nurse turnover affected half of the intervention practices, possibly contributing to reduced numbers of health checks and increased delays between the health check and follow up. Initial qualitative data also suggests that nurse turnover may have led to delays with patients getting appointments and some disengagement and dissatisfaction among patients with the intervention. We are unclear what this level of turnover represents although nurse retention is a widespread problem in Australia (23) and low satisfaction has been associated with poor retention among Australian PNs (24).

The positioning of PNs within general practice appears to have influenced, at least in part, the enthusiasm and willingness of some to participate fully in the HeLP GP intervention. While PNs were frequently autonomous in their daily work, we observed a general lack of practice-based support for them related to intervention delivery. Disorganised, dynamic environments and lack of strong leadership or 'top down' decision making were observed in some practices and this potentially contributed to a reduced sense of teamwork or lack of acknowledgement for the PN. 
Despite efforts at practice recruitment to speak to and engage as many PNs as possible, they were frequently excluded from the decision to participate in the trial. This has been shown in other nurse led weight management programs to cause poor ownership and commitment (12). Additionally, the high level of turnover meant that many PNs inherited a role they were not prepared for and possibly did not want. PNs took on the bulk of the trial responsibilities, including in many cases, coordination of the receptionists and the GPs. In part, intervention success was therefore reliant on the individual's level of interest, expertise, and availability. Many GPs and PNs expressed concern at the outset about the possible impost on PNs. Around $65 \%$ of PNs were employed in the practice part-time and it appeared that many PNs were under-resourced to take on the extra work. Although some remuneration was provided, PNs could not bill their time to Medicare.

The general profile of PNs in Australia is one of varying levels of experience and training, compounded by the geographic location in which they work, the type of population they see, and the parameters put on their role by the employing practice $(24,25)$. PNs are constrained by the lack of post graduate opportunities for training relevant to general practice (26) and roles geared towards procedural tasks that are reimbursed through Medicare (27). In this trial, we observed substantially different individual levels of proficiency with intervention tasks, possibly due to variations in skill levels, interest and/or priority. This has similarly been reported in other obesity trials (28). Specific intervention training was provided in on-line format and monitoring was done to ensure that all PNs completed all three modules, and this was reinforced in the facilitation sessions. Basic clinical tasks inherent in the health check (BP, BMI, WC etc) and follow up of patients did not produce notable difficulties for PNs. However, we observed some difficulty/reluctance by PNs to get patients to attend the health check or to provide sufficient contact attempts to get patients through the intervention. Referral to the telephone coaching also did not prove problematic but we noted low confidence among some PNs with instructing their patients on mysnapp. It may have been this lack of confidence which resulted in low willingness to help patients to install the app, or to teach them to use it.

The HeLP GP intervention aimed to assess the value, sustainability, and scalability of a nurse-led model to provide management to overweight and obese patients. The challenges identified have implications for the value of this model going forward. The model used in one NSW practice where additional nurses were paid to conduct the health checks for enrolled patients proved more efficient and generally more successful than using the practice PN. While this may be a suitable solution for short term research, it is not a low cost, easy to implement, scalable or sustainable model for general practice. Our experience suggests that undertaking a health check and undertaking lifestyle management for this population is a relatively complex organisational activity and PNs may need more than just training to integrate this into daily workflow. This trial incorporated a series of at least three facilitation sessions to each PN to help build confidence and provide support around the intervention tasks. This included options for referral to a lifestyle app and telephone coaching to supplement their role. While the facilitation sessions were well accepted and valued by PNs, they did not result in a consistent level of intervention for all PNs. The incorporation of the lifestyle app and the telephone coaching aimed to support the PNs and provide additional resources to help patients meet their goals. PNs did not baulk at coaching referral but they experienced barriers with using the app as a support tool. The reasons for this should be further explored through qualitative data.

\section{Strengths and Limitations}

This research identified organisational factors influencing the implementation of a general practice-based weight management intervention. Use of a recognised organisational readiness framework has provided some useful and relevant considerations. The decision to assess organisational readiness was largely post-hoc and outside of the main trial objectives. It did however provide an additional avenue by which to build context around the trial findings, particularly the structures, organisation and funding which are important implementation considerations in this setting.

Completion of the organisational readiness framework was conducted retrospectively. Quantitative data were obtained from a range of trial instruments and qualitative documentation was collected by the ROs and facilitators through their interactions with the practices. The mixing of this data, plus the group workshop to populate the framework, was done post intervention. This, however provided a broader and richer understanding of the factors affecting organisational readiness within the trial, as well as substantiating the views of individuals. It is possible that some bias may have been introduced as researchers may have already developed ideas and beliefs (positive or negative) about individual practices that were reflected in their responses at this point. Also, the assessment of organisational readiness reflects the period of the trial and therefore whatever cultural or workforce situation was present within the general practice at that time. As general practices are fluid entities, this may be an indication only of their state of organisational readiness.

\section{Conclusion}

An organisational readiness checklist is a valuable tool to employ so specific capacity issues can be addressed prior to commencing research. Within general practice this should assess the capacity of the individual responsible for the implementation, and the practice generally. This trial showed a lack of general 'readiness' inherent in the role of our participants. If PNs are to fulfil their potential in supporting patients to reduce risk and adopt healthier life choices, our study indicates that more could be done to improve their workforce positioning and remuneration which may improve continuity of care, retention, and individual motivation.

\section{Abbreviations}

BMI - Body Mass Index

DCP - Doctors Control Panel

GP - General Practice

GPs - General Practitioners 
LGA - Local Government Areas

NSW - New South Wales

PM - Practice Manager

PNs - Practice Nurses

PHN - Primary Health Network

SA - South Australia

SEIFA - Socioeconomic Index for Areas

UoA - University of Adelaide

UNSW - University of New South Wales

WC - Waist Circumference

\section{Declarations}

\section{Ethics approval and consent to participate.}

Ethical approval was obtained for the conduct of this trial from the University of New South Wales Human Research Ethics Committee (UNSW HREC HC174).

\section{Consent for publication.}

Not applicable

\section{Availability of data and materials.}

The datasets used and/or analysed during the current study are available from the corresponding author on reasonable request. Data relevant to this study have been summarised and presented in this article and its supplementary files.

\section{Competing interests.}

The authors declare that they have no competing interests.

\section{Funding.}

This work is supported by the National Health and Medical Research Council (NHMRC) of Australia project grant number APP1125681 (2017).

\section{Authors' contributions}

SP coordinated the HeLP GP study, contributed to the design of the overall trial, and developed the concept for this sub-study. AT, SS and CM interfaced with practices, and collected and compiled research data used in this article including qualitative research and field notes. SP AT, SS and CM participated in the workshop where intervention practice data were mapped to the organisational readiness framework. MH, EDW and DN designed the HeLP GP trial and contributed to the development of this sub-study. SP drafted this article and all other authors contributed to the final version.

\section{Acknowledgements}

Further information about the trial group can be obtained from m.f.harris@unsw.edu.au. The Investigators of the Health e-Literacy for Prevention in General Practice (HeLP GP) Trial Group:

Professor Mark Harris (Chief Investigator and Study Lead) University of New South Wales, NSW, Australia.

Professor Nigel Stocks (Chief Investigator) The University of Adelaide, SA, Australia.

Professor Donald Nutbeam (Chief Investigator) The University of Sydney, NSW, Australia.

Professor Nicholas Zwar (Chief Investigator) Bond University, Qld, Australia.

Professor Jonathan Karnon (Chief Investigator) The University of Adelaide, SA, Australia.

Professor Elizabeth Denney-Wilson (Chief Investigator) The University of Sydney, NSW Australia.

Professor Siaw-Teng Liaw (Chief Investigator) University of New South Wales, NSW, Australia. 
Dr Annie Lau (Chief Investigator) Macquarie University, NSW, Australia.

Associate Professor Margo Barr (Chief Investigator) University of New South Wales, NSW, Australia

We also acknowledge the support of the South Western Sydney Primary Health Network, Adelaide Primary Health Network and Nepean Blue Mountains Primary Health Network who assisted with the recruitment of general practices to this trial. We also thank all the participating practices, staff and patients for their interest and contribution to this research.

\section{References}

1. World Health Organisation (WHO). Fact sheet on obesity and overweight [Available from: https://www.who.int/en/news-room/fact-sheets/detail/obesityand-overweight - accessed 8/4/2020.

2. Australian Bureau of Statistics (ABS). Australian Health Survey: first results, 2011-12. Canberra Australian Government; 2012.

3. Royal Australian College of General Practitioners (RACGP). Obesity Prevention and Management: Position Statement 2019.

4. Australian Institute of Health and Welfare. A picture of overweight and obesity in Australia 2017. Canbera: AlHW; 2017.

5. Gearon E, Backholer K, Lal A, Nusselder W, Peeters A. The case for action on socioeconomic differences in overweight and obesity among Australian adults: modelling the disease burden and healthcare costs. Aust N Z J Public Health. 2020;44:121-8.

6. Britt H, Miller GC, Henderson J, Bayram C, Harrison C, Valenti L, et al. General practice activity in Australia 2015-16. Sydney; 2016.

7. Ashman F, Sturgiss E, Haesler E. Exploring self-efficacy in Australian general practitioners managing patient obesity: A qualitative survey study. International Journal of Family Medicine. 2016.

8. Blackburn M, Stathi A, Keogh E, Eccleston C. Raising the topic of weight in general practice: perspectives of GPs and primary care nurses. BMJ Open 2015;5.

9. Hobbs M, Radley D. Obesogenic environments and obesity: a comment on 'Are environmental area characteristics at birth associated with overweight and obesity in school-aged children? Findings from the SLOPE (Studying Lifecourse Obesity PrEdictors) population-based cohort in the south of England'. BMC Med. 2020;18:59.

10. Grima M, Dixon JB, Obesity. Recommendations for management in general practice and beyond. Australian Family Physician. 2013;42(8).

11. Forgione N, Deed G, Kilov G, Rigas G. Managing Obesity in Primary Care: Breaking Down the Barriers. Advanced Therapies. 2018;35:191-98.

12. Counterweight Project Team. McQuigg M, Brown J, Broom J, Laws R, Reckless J, et al. Evaluation of the Counterweight Programme for obesity management in primary care: a starting point for continuous improvement British Journal of General Practice. 2008; 58(553):548-54.

13. Gray J, Hoon E, Haji Ali Afzali H, Spooner C, Harris MF, Karnon J. Is the Counterweight Program a feasible and acceptable option for structured weight management delivered by practice nurses in Australia? A mixed-methods study. Australian Journal of Primary Health. 2017;23:348-63.

14. Shea C, Jacobs SR, D.A E, Bruce K. Weiner B.J. Organizational readiness for implementingchange: a psychometric assessment of a new measure. Implementation Science. 2014;9(7).

15. Helfrich CD, Blevins D, Smith JL, Kelly A, Hogan TP, Hagedorn H, et al. Predicting implementation from organizational readiness for change: a study protocol. Implementation Science 2011;6(76).

16. Shaw RJ, Kaufman MA, Bosworth HB. Organisational factors associated with readiness to implement and translate a primary care based telemedicine behavioural program to improve blood pressure control: the HTN-IMPROVE study. Implementation Science. 2013;8(106).

17. Parker SM, Stocks N, Nutbeam D, Louise Thomas E, Denney-Wilson N, Zwar, et al. Preventing chronic disease in patients with low health literacy using eHealth and teamwork in primary healthcare: protocol for a cluster randomised controlled trial. BMJ Open 2018.

18. Australian Bureau of Statistics (ABS). [Available from: http://stat.data.abs.gov.au/Index.aspx?DataSetCode=ABS_SEIFA_LGA.

19. Haji Ali Afzali H, Karnon J, Beilby J, Gray J, Holton C, Banham D. Practice nurse involvement in general practice clinical care: policy and funding issues need resolution. Australian Health Review 2014.

20. Scaccia J, Cook B, Lamont A, Wandersam A, Castellow J, Katz J. Journal of Community Psychology A Practical Implementation Science Heuristic for Organisational Readines: R = MC2. 2015;43(4):484-501.

21. O'Cathain A, Murphy EJ. The quality of mixed methods studies in health services research. Journal of Health Services Research Policy. 2008;13(2):92-8.

22. NSW Agency for Clinical Innovation. Understanding and working with general practice. Sydney: NSW Agency for Clinical Innovation; 2015.

23. Dawson A, Stasa H, Roche M, Homer C, Duffield C. Nursing churn and turnover in Australian hospitals: nurses perceptions and suggestions for supportive strategies. BMC Nursing 2014;13(11).

24. Halcomb E, Bird S, Mcinnes S, Ashley C, Huckel K. Exploring job satisfaction and turnover intentions among general practice nurses in an Australian Primary Health Network. Journal of Nursing Management; 2020.

25. Australian College of Nursing. A new horizon for health service: Optimising advanced practice nursing. 2019.

26. Heywood T, Laurence C. The general practice nurse workforce. Estimating future supply. Australian Journal of General Practice 2018;47(11).

27. Henderson J, Koehne K, Verrall C, Gebbie K, Fuller J. How is Primary Health Care conceptualised in nursing in Australia? A review of the literature. Health Soc Care Community. 2014;22:337-51.

28. Campbell-Scherer DL, Asselin J, Osunlana AM, Ogunleye A, Fielding S, Anderson R, et al. Changing provider behaviour to increase nurse visits for obesity in family practice: the 5As Team randomized controlled trial. CMAJ Open. 2019;7(2):371-8.

Page 9/16 


\section{Tables}

Table 1. Commitment of the PN within the HeLP GP intervention.

\begin{tabular}{|c|c|}
\hline $\begin{array}{l}\text { Time } \\
\text { commitment }\end{array}$ & PN Task \\
\hline 60 minutes & $\begin{array}{l}3 \text { on-line training modules to assist PNs to deliver the intervention. Training covered guideline recommendations regarding diet and } \\
\text { physical activity, and modules on health literacy, weight management and motivational interviewing. Teach back tools were encouraged } \\
\text { within interactions to assess patient knowledge and understanding (health literacy). }\end{array}$ \\
\hline 2-3 hours & $\begin{array}{l}3 \text { facilitation visits provided by local PHN staff or a research nurse. These visits supported PNs to work with their patients on improved } \\
\text { health literacy, goal directions, and addressing challenges and difficulties as patients progressed through the trial. }\end{array}$ \\
\hline $\begin{array}{l}30-40 \\
\text { minutes per } \\
\text { patient }\end{array}$ & $\begin{array}{l}\text { Health check based on the 5As (Assess, Advise/Agree, Assist and Arrange). As well as doing basic biometric measurements, PNs used a } \\
\text { trial template to prompt discussions about weight management, diet, and physical activity and to set up mysnapp using each patient's } \\
\text { baseline measurements. This served as the basis for the personal goals set by the patients for the next } 6 \text { weeks. The PNs also provided } \\
\text { assisted referral to the Get Healthy telephone coaching service provided by the NSW government where patients could receive up to } 10 \\
\text { tailored coaching calls (https://www.gethealthynsw.com.au/). }\end{array}$ \\
\hline $\begin{array}{l}20 \text { minutes } \\
\text { per patient }\end{array}$ & $\begin{array}{l}\text { 6-week review at which weight, BMI and WC were re-measured and patient progress or difficulty was used to revise the patient's goals. The } \\
\text { PNs were expected to review the patient's experience of mysnapp and Get Healthy and schedule a further } 12 \text {-week review with the GP. }\end{array}$ \\
\hline
\end{tabular}

20 minutes PNs were expected to review the patient's experience of mysnapp and Get Healthy and schedule a further 12-week review with the GP.

Table 2. Organisational readiness components and sub-components. 


\section{MOTIVATION - The motivation to implement the intervention}

Compatibility

Was the intervention perceived as being consistent with existing values and beliefs belonging to the practice?

Complexity

Did the practice feel that the intervention was 'doable'

Priority

Was the intervention seen as a priority? Or was it low in priority?

\section{ORGANISATIONAL CAPACITY - The attributes associated with a functioning organisation}

Culture/Climate

Were the practice staff equally involved in decision making around involvement with the intervention?

Was there scope for practice staff (particularly PNs) to have ownership of their work? Including managing the intervention

Did staff at the practice generally express job satisfaction?

General Capacity/Resource Utilisation

Were there sufficient staffing available at the practice to participate?

Was PN turnover an issue for the practice?

Were there any notable issues related to space? Equipment or technical resources?

Leadership

Was there evidence of effective leadership within the practice?

\section{INTERVENTION SPECIFIC CAPACITY - The human, technical and fiscal conditions that are important to implement the intervention}

Supportive Climate

Intervention specific resources

\section{Level of PN receptiveness}

The general level of support for the intervention by staff in the practice (i.e. PNs, GPs, receptionists, Practice Manager)?

Uptake of trial resources?

Time and resource issues expressed/experienced by the practice?

Difficulty with systems (DCP, health check templates, mysnapp, Get Healthy)?

Capacity/Enthusiasm of PN for training?

Level of PN engagement with the facilitation sessions

PN specific skills, knowledge, and abilities to deliver the intervention

Level of PN confidence with conducting health checks

Level of PN confidence to work with the patient with mysnapp?

Level of PN confidence to refer patients to Get Healthy

Level of PN success with delivering health checks

Evidence of a 'champion' at the practice?

Extent to which the intervention was supported/hindered by the practice management?

PNs feeling supported to undertake the tasks related to the intervention?

Source: Jonathan P. Scaccia, et al. (2015). "A Practical Implementation Science Heuristic for Organisational Readiness: R = MC2." Journal of Community Psychology_43(4): 484-501.

Table 3. Baseline characteristics of all practices.

\begin{tabular}{|c|c|c|c|c|c|c|}
\hline & \multicolumn{2}{|c|}{ Intervention } & \multicolumn{2}{|l|}{ Control } & \multicolumn{2}{|l|}{ TOTAL } \\
\hline No of Practices & \multicolumn{2}{|l|}{11} & \multicolumn{2}{|l|}{11} & \multicolumn{2}{|l|}{22} \\
\hline NSW & \multicolumn{2}{|l|}{8} & \multicolumn{2}{|l|}{7} & \multicolumn{2}{|l|}{15} \\
\hline SA & \multicolumn{2}{|l|}{3} & \multicolumn{2}{|l|}{4} & \multicolumn{2}{|l|}{7} \\
\hline \multirow[t]{3}{*}{ Size of practices } & \multicolumn{2}{|c|}{5 practices $<5$ GPs } & \multicolumn{4}{|c|}{5 practice $<5$ GPs } \\
\hline & \multicolumn{2}{|c|}{6 practices $>=5 \mathrm{GPs}$} & \multicolumn{4}{|c|}{6 practices $>=5 \mathrm{GPs}$} \\
\hline & $\mathrm{N}$ & $\%$ & $\mathrm{~N}$ & $\%$ & $\mathrm{~N}$ & $\%$ \\
\hline Participating GPs/all GPs in practices & $17 / 54$ & $31.5 \%$ & $24 / 63$ & $38.1 \%$ & $41 / 117$ & $35.0 \%$ \\
\hline Participating PNs/all PNs in practices & $19 / 24$ & $79.2 \%$ & $18 / 22$ & $81.8 \%$ & $37 / 46$ & $80.4 \%$ \\
\hline Total Participants & $36 / 78$ & $46.2 \%$ & $42 / 85$ & $49.4 \%$ & $78 / 163$ & $48.1 \%$ \\
\hline
\end{tabular}


Table 4. Baseline characteristics of all providers.

\begin{tabular}{|c|c|c|c|c|c|c|c|}
\hline & GPs & $\%$ & PNS (N) & $\%$ & & $\%$ & \\
\hline & $(\mathrm{N})$ & & & & TOTAL (N) & & P-Value[1] \\
\hline \multicolumn{8}{|l|}{ State } \\
\hline NSW & 26 & $63.4 \%$ & 24 & $64.9 \%$ & 50 & $64.1 \%$ & \\
\hline SA & 15 & $36.6 \%$ & 13 & $35.1 \%$ & 28 & $35.9 \%$ & \\
\hline TOTAL & 41 & & 37 & & 78 & & \\
\hline Intervention & 17 & $41.5 \%$ & 19 & $51.4 \%$ & 36 & $46.2 \%$ & \\
\hline Control & 24 & $58.5 \%$ & 18 & $48.6 \%$ & 42 & $53.8 \%$ & \\
\hline \multicolumn{8}{|l|}{ Gender } \\
\hline Female & 20 & & 34 & & 54 & & \\
\hline Male & 21 & & 3 & & 24 & & \\
\hline \multicolumn{8}{|l|}{ Age Group } \\
\hline $20-34$ & 6 & $14.6 \%$ & 20 & $54.1 \%$ & 26 & $33.3 \%$ & \\
\hline $35-44$ & 10 & $24.4 \%$ & 6 & $16.2 \%$ & 16 & $20.5 \%$ & \\
\hline $45-54$ & 9 & $22.0 \%$ & 8 & $21.6 \%$ & 17 & $21.8 \%$ & \\
\hline $55-64$ & 14 & $34.1 \%$ & 2 & $5.4 \%$ & 16 & $20.5 \%$ & \\
\hline $65+$ & 2 & $4.9 \%$ & 0 & $0.0 \%$ & 2 & $2.6 \%$ & \\
\hline \multicolumn{8}{|l|}{ Work Status } \\
\hline Full time & 35 & $85.4 \%$ & 13 & $35.1 \%$ & 48 & $61.5 \%$ & \\
\hline Part time & 6 & $14.6 \%$ & 24 & $64.9 \%$ & 30 & $38.5 \%$ & \\
\hline Years of work in General Practice & Mean & SD & Mean & SD & Mean & SD & \\
\hline Years in general practice & 14.5 & 12.3 & 4 & 4.5 & 9.6 & 10.8 & \\
\hline Missing (6 cases) & 3 & & 3 & & & & \\
\hline Years in this practice & 10.1 & 12.1 & 2.2 & 3.0 & 6.3 & 9.7 & \\
\hline Missing (1 case) & 1 & & 0 & & & & \\
\hline Use of Apps/websites with patients & $\mathbf{N}$ & $\%$ & $\mathbf{N}$ & $\%$ & $\mathbf{N}$ & $\%$ & \\
\hline Never/Rarely & 19 & $46.3 \%$ & 18 & $48.6 \%$ & 37 & $47.4 \%$ & \\
\hline Sometimes/half the time & 16 & $39 \%$ & 10 & $27 \%$ & 26 & $33.3 \%$ & \\
\hline Often/usually/always & 6 & $14.6 \%$ & 9 & $25 \%$ & 15 & $19.2 \%$ & NS* \\
\hline \multicolumn{8}{|c|}{ Confidence showing patient how to use apps } \\
\hline Not at all confident & 7 & $17.1 \%$ & 2 & $5.6 \%$ & 9 & $11.7 \%$ & \\
\hline Minimally confident & 10 & $24.4 \%$ & 8 & $22.2 \%$ & 18 & $23.4 \%$ & \\
\hline Somewhat confident & 15 & $36.6 \%$ & 7 & $19.4 \%$ & 22 & $28.6 \%$ & \\
\hline Moderately confident & 7 & $17.1 \%$ & 11 & $30.6 \%$ & 18 & $23.4 \%$ & \\
\hline Very confident & 2 & $4.9 \%$ & 8 & $22.2 \%$ & 10 & $13.0 \%$ & $P=0.041^{*}$ \\
\hline
\end{tabular}

Table 5. Organisational Readiness Domains 


\begin{tabular}{|c|c|c|c|c|c|c|c|c|c|}
\hline & Component & NSW 01 & NSW 02 & NSW 03 & NSW 04 & NSW 05 & NSW 06 & NSW 08 & SA 01 \\
\hline \multicolumn{10}{|c|}{ GENERAL CHARACTERISTICS } \\
\hline \multicolumn{2}{|c|}{ Size of practice (No. of GPs) } & $<5$ & $\geq 5$ & $<5$ & $<5$ & $<5$ & $<5$ & $<5$ & $\geq 5$ \\
\hline \multicolumn{2}{|l|}{ No. of PNs } & 1 & 2 & 4 & 1 & 4 & 1 & 3 & 1 \\
\hline \multicolumn{2}{|c|}{ No. of participating PNs } & 1 & $1-2$ & 1 & 1 & 1 & 1 & 1 & 1 \\
\hline \multicolumn{2}{|c|}{ No. of patients recruited } & 50 & 27 & 51 & 3 & 9 & 4 & 1 & 11 \\
\hline \multicolumn{2}{|c|}{ No. of health checks conducted } & 21 & 15 & 24 & 0 & 2 & 2 & 1 & 10 \\
\hline MOTIVATION & Component & & & & & & & & \\
\hline Compatibility & $\begin{array}{l}\text { Intervention perceived } \\
\text { as consistent with } \\
\text { existing values and } \\
\text { beliefs }\end{array}$ & Unclear & Yes ${ }^{\star \star \star}$ & Unclear & Unclear & Unclear & Unclear & Unclear & Yes $^{\star \star \star}$ \\
\hline Complexity & $\begin{array}{l}\text { Practice felt the } \\
\text { intervention was } \\
\text { 'doable' }\end{array}$ & Yes ${ }^{\star \star \star}$ & Yes ${ }^{\star \star \star}$ & Yes ${ }^{\star \star \star}$ & Yes ${ }^{\star \star \star}$ & Yes ${ }^{\star \star \star}$ & Unclear & Yes $^{\star \star \star}$ & Yes ${ }^{\star \star \star}$ \\
\hline Priority & $\begin{array}{l}\text { Priority of HeLP GP } \\
\text { intervention }\end{array}$ & 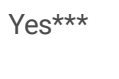 & Yes ${ }^{\star \star \star}$ & No* & 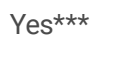 & Yes ${ }^{\star \star \star}$ & No* & No* & $\mathrm{No}^{*}$ \\
\hline $\begin{array}{l}\text { ORGANISATIONAL } \\
\text { CAPACITY }\end{array}$ & Component & & & & & & & & \\
\hline \multirow[t]{3}{*}{ Culture/Climate } & $\begin{array}{l}\text { Practice staff (PNs, } \\
\text { PMs, Reception, GPs) } \\
\text { are equally involved in } \\
\text { decision making } \\
\text { around involvement } \\
\text { with the intervention? }\end{array}$ & No* & No* & No* & $\mathrm{No}^{*}$ & $\mathrm{No}^{\star}$ & $\mathrm{No}^{*}$ & No* & Yes $^{\star \star \star}$ \\
\hline & $\begin{array}{l}\text { Scope for practice } \\
\text { staff (particularly PNs) } \\
\text { to have ownership of } \\
\text { their role/participation } \\
\text { in the intervention? }\end{array}$ & 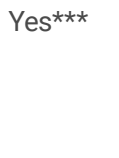 & Yes $* \star \star$ & 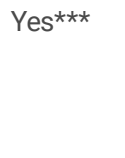 & Yes $* \star \star$ & Yes $* \star \star$ & Yes $* \star \star$ & Yes $* \star \star$ & 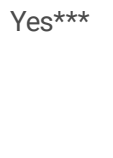 \\
\hline & $\begin{array}{l}\text { Expression of job } \\
\text { satisfaction among } \\
\text { practice staff }\end{array}$ & Unclear & Yes ${ }^{\star \star \star}$ & Yes ${ }^{\star \star \star}$ & Yes ${ }^{\star \star \star}$ & No* & Unclear & Unclear & Unclear \\
\hline \multirow[t]{3}{*}{$\begin{array}{l}\text { General } \\
\text { Capacity/Resource } \\
\text { Utilisation }\end{array}$} & $\begin{array}{l}\text { Were there sufficient } \\
\text { staffing available at } \\
\text { the practice to } \\
\text { participate? }\end{array}$ & No* & Yes ${ }^{\star \star \star}$ & $\mathrm{No}^{*}$ & Yes $* \star \star$ & Yes $* \star \star$ & Yes ${ }^{\star \star \star}$ & Yes $* \star \star$ & Yes $* \star \star$ \\
\hline & PN turnover & No* & Yes ${ }^{\star \star \star}$ & Yes $^{\star \star \star}$ & $\mathrm{No}^{\star}$ & 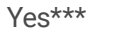 & $\mathrm{No}^{*}$ & 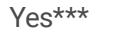 & No* \\
\hline & $\begin{array}{l}\text { Notable practice } \\
\text { issues related to } \\
\text { space, equipment or } \\
\text { technical resources? }\end{array}$ & $\mathrm{No}^{*}$ & Yes ${ }^{\star \star \star}$ & $\mathrm{No}^{*}$ & $\mathrm{No}^{*}$ & $\mathrm{No}^{*}$ & $\mathrm{No}^{*}$ & No* & $\mathrm{No}^{*}$ \\
\hline Leadership & $\begin{array}{l}\text { Was there evidence of } \\
\text { effective leadership } \\
\text { within the practice }\end{array}$ & No* & Yes ${ }^{\star \star \star}$ & Yes $* \star \star$ & Yes $* \star \star$ & Yes $^{\star \star \star}$ & 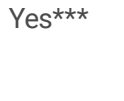 & $\mathrm{No}^{\star}$ & Yes ${ }^{\star \star \star}$ \\
\hline $\begin{array}{l}\text { INTERVENTION } \\
\text { SPECIFIC } \\
\text { CAPACITY }\end{array}$ & Component & & & & & & & & \\
\hline \multirow[t]{2}{*}{ Supportive Climate } & $\begin{array}{l}\text { Level of PN } \\
\text { receptiveness }\end{array}$ & Medium & Medium & Medium & $\mathrm{High}^{\star \star \star}$ & $\mathrm{High}^{\star \star \star \star}$ & Low* & Low* & $\mathrm{High}^{\star \star \star *}$ \\
\hline & $\begin{array}{l}\text { General support for the } \\
\text { intervention by staff } \\
\text { other than the PN in } \\
\text { the practice (i.e. } \\
\text { receptionists; PM)? }\end{array}$ & Medium & $\mathrm{High}^{\star \star \star}$ & Medium & $\mathrm{High}^{\star \star \star}$ & Medium & Medium & Medium & Medium \\
\hline \multirow[t]{2}{*}{$\begin{array}{l}\text { Intervention } \\
\text { specific resources }\end{array}$} & $\begin{array}{l}\text { Uptake of trial } \\
\text { resources }\end{array}$ & Medium & $\mathrm{High}^{\star \star \star \star}$ & $\mathrm{High}^{\star \star \star \star}$ & $\mathrm{High}^{\star \star \star \star}$ & $\mathrm{High}^{\star \star \star}$ & Medium & Low* & Low* \\
\hline & $\begin{array}{l}\text { Time and resource } \\
\text { issues } \\
\text { expressed/experienced } \\
\text { by the practice? }\end{array}$ & $\mathrm{No}^{*}$ & Yes ${ }^{\star \star \star}$ & Yes ${ }^{\star \star \star}$ & $\mathrm{No}^{\star}$ & $\mathrm{No}^{*}$ & Yes ${ }^{\star \star \star}$ & $\mathrm{No}^{*}$ & $\mathrm{No}^{\star}$ \\
\hline
\end{tabular}




\begin{tabular}{|c|c|c|c|c|c|c|c|c|c|}
\hline & Component & NSW 01 & NSW 02 & NSW 03 & NSW 04 & NSW 05 & NSW 06 & NSW 08 & SA 01 \\
\hline & $\begin{array}{l}\text { Difficulties with } \\
\text { systems? (DCP, health } \\
\text { check templates, } \\
\text { mysnapp, Get Healthy) }\end{array}$ & Yes $* * \star$ & Yes*** & 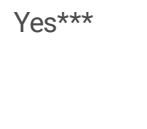 & Yes*** & Yes*** & Yes $* * \star$ & Yes*** & Yes*** \\
\hline & $\begin{array}{l}\text { Capacity/Enthusiasm } \\
\text { of PN for training? }\end{array}$ & Medium & Medium & Medium & Medium & Low* & Medium & Low* & Medium \\
\hline & $\begin{array}{l}\text { Level of PN } \\
\text { engagement with the } \\
\text { facilitation sessions }\end{array}$ & High*** & Med-High & $\mathrm{High}^{\star * \star *}$ & NA & Medium & 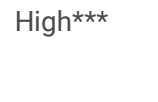 & Medium & Medium \\
\hline \multirow{4}{*}{$\begin{array}{l}\text { PN skills, } \\
\text { knowledge, and } \\
\text { abilities to deliver } \\
\text { the intervention }\end{array}$} & $\begin{array}{l}\text { Level of PN confidence } \\
\text { with conducting health } \\
\text { checks }\end{array}$ & High*** & Medium & High*** & NA & $\mathrm{High}^{\star \star \star}$ & High*** & 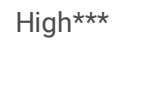 & $\mathrm{High}^{\star \star \star}$ \\
\hline & $\begin{array}{l}\text { Level of PN confidence } \\
\text { to work with the } \\
\text { patient with mysnapp? }\end{array}$ & Low* & 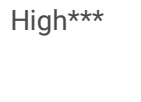 & Medium & NA & Medium & $\mathrm{High}^{\star \star \star}$ & Medium & 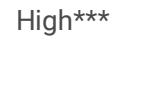 \\
\hline & $\begin{array}{l}\text { Level of PN confidence } \\
\text { to refer patients to Get } \\
\text { Healthy }\end{array}$ & $\mathrm{High}^{\star \star \star \star}$ & $\mathrm{High}^{\star \star \star *}$ & $\mathrm{High}^{\star * \star *}$ & NA & $\mathrm{High}^{\star \star \star *}$ & $\mathrm{High}^{\star * \star}$ & $\mathrm{High}^{\star * *}$ & $\mathrm{High}^{\star \star \star *}$ \\
\hline & $\begin{array}{l}\text { Level of PN success } \\
\text { with delivering health } \\
\text { checks }\end{array}$ & $\mathrm{High}^{\star \star \star}$ & Medium & 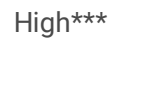 & NA & 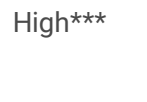 & $\mathrm{High}^{\star \star \star}$ & 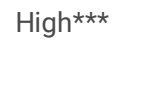 & 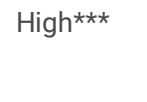 \\
\hline $\begin{array}{l}\text { Program } \\
\text { champion or driver }\end{array}$ & $\begin{array}{l}\text { Was there a } \\
\text { 'champion' at the } \\
\text { practice? }\end{array}$ & $\mathrm{No}^{*}$ & No* & No* & $\mathrm{No}^{*}$ & No* & No* & $\mathrm{No}^{\star}$ & No* \\
\hline \multirow[t]{2}{*}{$\begin{array}{l}\text { Implementation } \\
\text { support }\end{array}$} & $\begin{array}{l}\text { To what extent was } \\
\text { the intervention } \\
\text { supported or hindered } \\
\text { by the practice } \\
\text { management? }\end{array}$ & $\begin{array}{l}\text { Well- } \\
\text { supported } \\
\star \star \star\end{array}$ & $\begin{array}{l}\text { Well- } \\
\text { supported } \\
\star \star \star\end{array}$ & $\begin{array}{l}\text { Well- } \\
\text { supported } \\
\star \star *\end{array}$ & $\begin{array}{l}\text { Well- } \\
\text { supported } \\
\star \star \star\end{array}$ & $\begin{array}{l}\text { Well- } \\
\text { supported } \\
\star \star \star\end{array}$ & Supported & Supported & Supported \\
\hline & $\begin{array}{l}\text { Did PNs feel supported } \\
\text { to undertake the } \\
\text { intervention tasks? }\end{array}$ & No* & 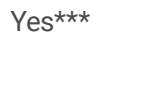 & No* & No* & No* & No* & No* & 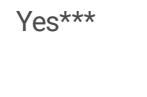 \\
\hline
\end{tabular}

*** Positive result *Negative result

NA - No patients recruited.

Table 6. List of trial resources.

\begin{tabular}{|c|c|c|c|}
\hline & Resource & Source & Audience \\
\hline \multirow[t]{2}{*}{ Facilitation } & PHN flip book guide to facilitation visits & Microsoft Teams & Facilitator \\
\hline & Intervention facilitator diary of practice visits & Microsoft Teams & Facilitator \\
\hline Training & Modules 1, 2, 3 & Smart Sparrow educational platform via provider website & PN, GP \\
\hline \multirow[t]{9}{*}{ Intervention } & Overview of the HeLP-GP Clinical Intervention & $\begin{array}{l}\text { Microsoft Teams, practice package (hard copy), provider } \\
\text { website }\end{array}$ & Practice \\
\hline & DCP Instructions & Provider website & Practice \\
\hline & Protocol Summary for GPs & $\begin{array}{l}\text { Microsoft Teams, practice package (hard copy), provider } \\
\text { website }\end{array}$ & Practice \\
\hline & $\begin{array}{l}\text { Flow chart/flip book to support nurses to deliver health } \\
\text { checks/follow up }\end{array}$ & $\begin{array}{l}\text { Microsoft Teams, practice package (hard copy), provider } \\
\text { website }\end{array}$ & PN \\
\hline & Health Check Template & Practice package (hard copy), Clinical Software & PN \\
\hline & mysnapp information for practices - troubleshooting & Practice package (hard copy), patient website & $\begin{array}{l}\text { PN, } \\
\text { Patient }\end{array}$ \\
\hline & mysnapp information for practices iOS and Android & Practice Package (hard copy), Patient Website & $\begin{array}{l}\text { PN, } \\
\text { Patient }\end{array}$ \\
\hline & mysnapp video & Teams, practice package, patient website & $\begin{array}{l}\text { PN, } \\
\text { Patient }\end{array}$ \\
\hline & Get Healthy referral materials & Teams, practice package & PN \\
\hline
\end{tabular}


Table 7. Facilitation summary.

\begin{tabular}{|llll|}
\hline $\begin{array}{l}\text { NSW (n=8 intervention practices) } \\
\text { SA ( } \mathrm{n}=3 \text { intervention practices) }\end{array}$ & & & \\
\hline Average number of visits & & & \\
& NSW (8 practices) & Range 1-7 \\
& SA (3 practices) & Range 1-8 \\
\hline & All practices & 4.7 \\
\hline Average time spent (minutes) per visit: & Scheduling & 9 \\
\hline & Travel & 67 \\
\hline & Waiting & 14 \\
\hline & Facilitation & 48 \\
\hline & FV 1 only & 40 \\
\hline & FV 2 only & 98 \\
\hline & FV 3 only & 34 \\
\hline
\end{tabular}

\section{Figures}

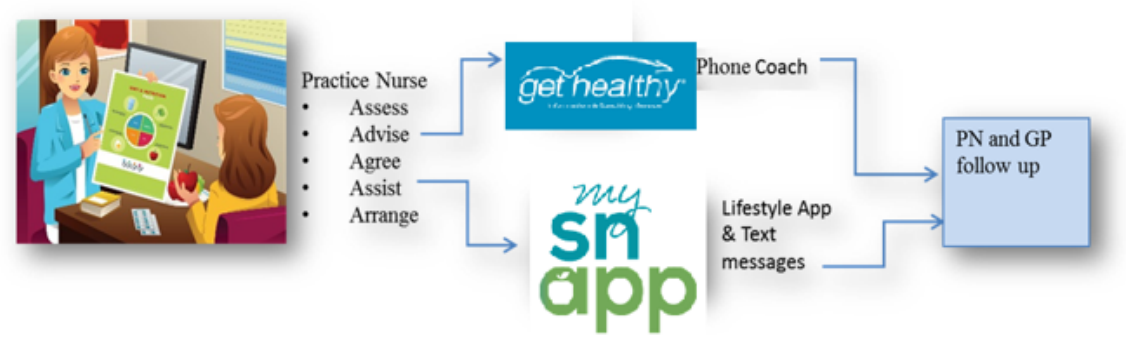

\section{Figure 1}

The intervention was a PN led model of health assessment combined with mHealth (mysnapp lifestyle app) and telephone coaching (Get Healthy). Access to the app and the coaching were facilitated by the PNs and both the PNs and GPs conducted patient follow up ( 6 and 12 weeks respectively) (Figure 1 ). The trial sought to help overweight or obese patients to make positive lifestyle changes to diet and physical activity and to assess the value, sustainability, and scalability of this nurse-led model. 


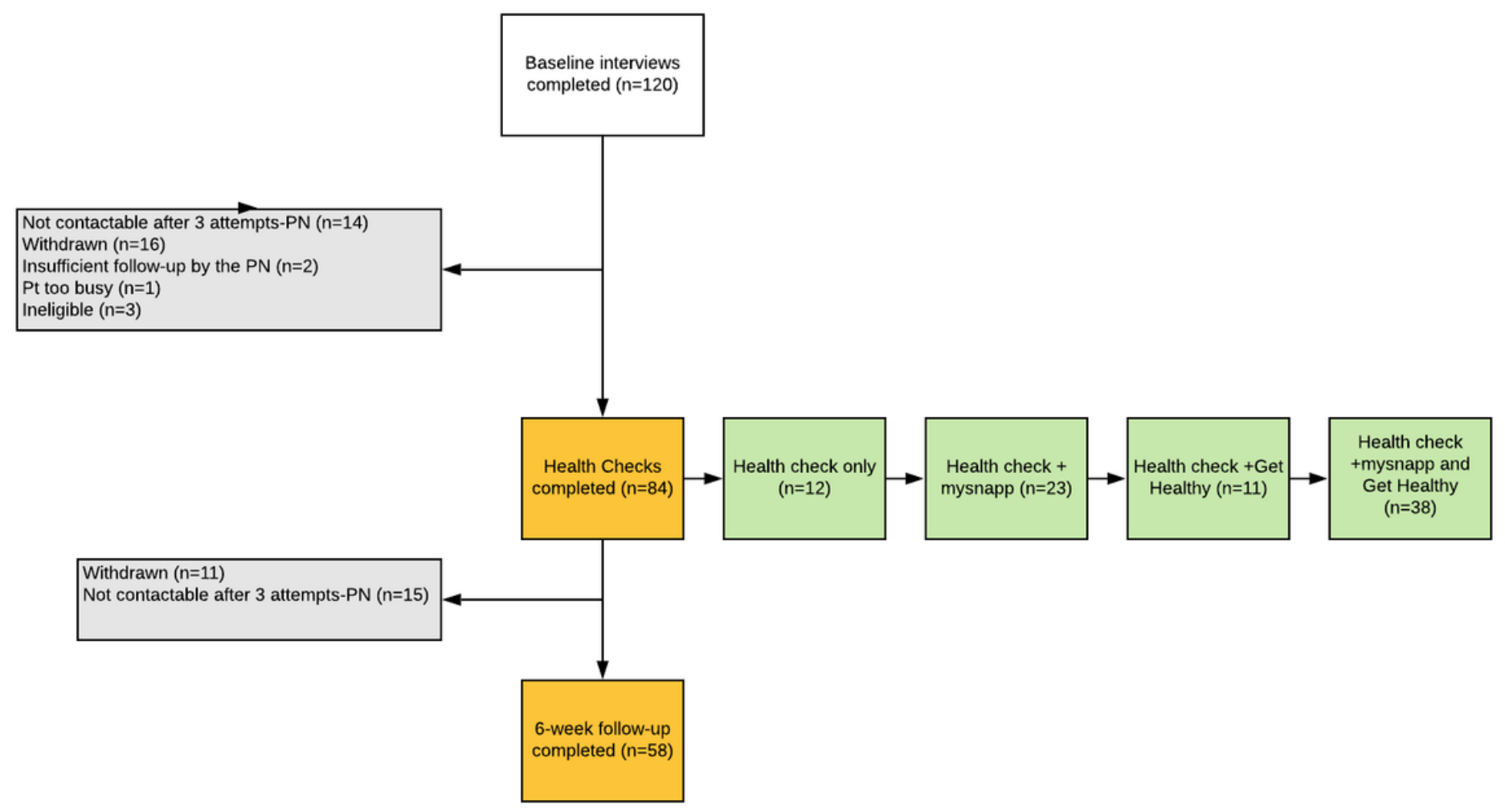

Figure 2

The HeLP GP intervention had three components that PNs could offer to their patients. Feedback and observation indicated that PNs were comfortable with the clinical content of the health check as this largely aligned with their normal work. Despite this, ROs and facilitators experienced significant ambivalence on the part of the PNs to conduct the health checks, noting a mix of disinterest, reluctance, and a lack of confidence by PNs to engage with their patients throughout the intervention. On-line training appeared well received, providing obesity education and tools and instruction to implement the intervention. PNs however, demonstrated variable success with delivering the health checks to consenting patients. One Sydney practice failed to successfully recruit any patients and hence no health checks were conducted at this site. In total 84/120 (70\%) consenting patients underwent a health check and completed the baseline assessment. At the point of health check, 16 patients withdrew, 14 patients could not be contacted after three attempts by the PN, and 3 patients were found to be ineligible. The health check was not completed for 2 patients who received insufficient follow up by the PN and 1 patient who was too busy to attend. Similarly, PNs struggled to complete the patient follow-up with 58/84 (69\%) patients being seen for the 6-week review (Figure 2). 